\title{
Developmental dysplasia of the hip: why are we still operating on them? A plea for institutional newborn clinical screening
}

\author{
Wu Chean $\underline{\mathrm{Lee}}^{1}$, MBChB, MRCs, Sumanth Kumar $\underline{\mathrm{Gera}}^{2}$, MBBS, MS, Arjandas $\underline{\text { Mahadev}}{ }^{2}$, MBBS, FAMS
}

INTRODUCTION Developmental dysplasia of the hip (DDH) is a common orthopaedic condition at birth. Non-surgical management with the Pavlik harness can effectively treat DDH in the newborn by providing an early clinical diagnosis, but open surgeries continue to be performed. We aimed to elucidate the reasons for this.

METHODS A retrospective review was performed of all open surgeries related to DDH from 2006 to 2016 . Patients were either born at our institution (Group 1) or outside of it (Group 2). All Group 1 newborns were routinely screened for DDH at birth.

RESULTS 27 patients (Group 1: $n=5$, Group 2: $n=22$ ) presented at age $25 \pm 19$ months. Left-sided DDH $(n=21,77.8 \%)$ and female infants $(n=22,81.5 \%)$ were more common. The mean age at surgery was $40 \pm 31$ months. The most commonly performed procedure was soft tissue release open reduction with acetabuloplasty $(n=20,74.1 \%)$. Gender, site, median age at presentation and at surgery, and prevalence of risk factors were similar for both groups. Both groups were mostly made up of late presenters ( $>3$ months; $p=0.34$ ). A few patients had undergone prior treatment $(p=0.64)$. Newborn screening was the only significantly different variable between the groups $(p<0.01)$.

CONCLUSION Lack of institutionalised newborn clinical screening appears to be the root cause of late presentation of DDH leading to open surgery for its management. We recommend quality institutionalised newborn clinical screening to reduce the number of late presentations.

Keywords: developmental dysplasia of the hip, newborn screening, open reduction

\section{INTRODUCTION}

Developmental dysplasia of the hip $(\mathrm{DDH})$ is one of the commonest hip abnormalities found at birth. ${ }^{(1)}$ It is a wellestablished risk factor for early-onset osteoarthritis of the hip and the resultant arthroplasty. ${ }^{(1)}$

Screening at birth using the Ortolani test ${ }^{(2)}$ and Barlow test, ${ }^{(3)}$ henceforth to be referred to as 'newborn clinical screening' in this study, allows for the early diagnosis of $\mathrm{DDH}$, and has excellent sensitivity and specificity in experienced hands. ${ }^{(4,5)}$ The success rate of intervening early with conservative treatment, such as the Pavlik harness, has been reported to be as high as $96.7 \%$, with the rate of avascular necrosis as low as $0 \% .{ }^{(6)}$ Such early diagnosis and conservative treatment has been reported to reduce the rate of surgery required for $\mathrm{DDH}$, or even obviate the need for invasive procedures. ${ }^{(7)}$

Rates of all types of open surgery for DDH have been reported to be reduced significantly by effective institutional newborn clinical screening programmes. ${ }^{(8)}$ At our institution, all newborns are screened by neonatology physicians and referred to orthopaedics upon clinical suspicion of DDH (Fig. 1). Clinical suspicion of DDH at birth refers to anything other than a normal finding of the hips at birth during clinical screening, such as a positive Ortolani or Barlow test, hip laxity and hip clicks. A formal diagnosis is only given if the referred orthopaedic surgeon finds a positive Ortolani or Barlow test. In the absence of a positive Ortolani or Barlow test, but with findings of hip laxity and hip clicks as well as the presence of risk factors on neonatal clinical screening, a hip ultrasonography is performed at age 6-8 weeks and the child is referred to orthopaedics upon findings of dysplasia. We define hip laxity as an Ortolani or Barlow test that is not clinically obvious yet not entirely normal. Treatment with the Pavlik harness is then undertaken according to the algorithm in Fig. 1.

Our institution sees an annual average of 12,000 live births. A preliminary internal five-year review of the institution's DDH newborn clinical screening and treatment protocol revealed that 177 orthopaedics referrals were made for suspected $\mathrm{DDH}$, with 124 patients eventually being diagnosed with $\mathrm{DDH}$. Of these, only one patient required open reduction. Despite the purported success of early diagnosis and treatment, we continue to frequently perform surgeries related to DDH on an anecdotal basis.

Sanghrajka et $\mathrm{al}^{(8)}$ previously found that open surgeries for DDH continued to be performed due to late presentation secondary to failure at the level of newborn screening. Their study was limited to a five-year review in a Caucasian population and the findings have yet to be replicated elsewhere. Hence, the present study aimed to review open surgeries for $\mathrm{DDH}$ at our institution with regard to the indications for surgery. We hypothesised that a lack of institutionalised newborn clinical screening is the root cause of open surgery for DDH. Our findings may help to guide future improvement in the management of $\mathrm{DDH}$ and reduce the need for preventable open surgery.

${ }^{1}$ Department of Orthopaedic Surgery, Singapore General Hospital, 2Department of Orthopaedic Surgery, KK Women's and Children's Hospital, Singapore 


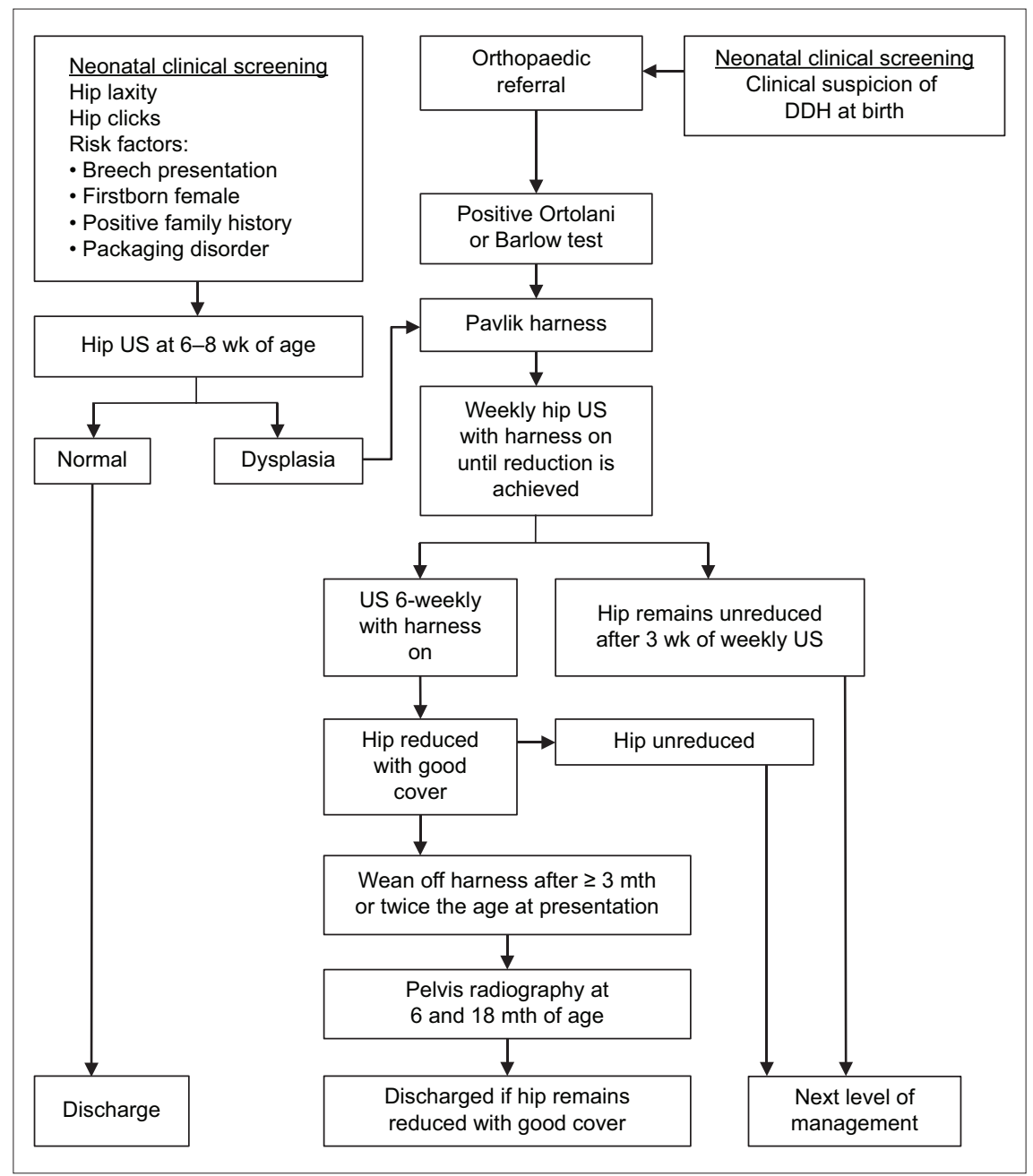

Fig. 1 Flowchart shows the institutional neonatal clinical screening for developmental dysplasia of the hip (DDH). US: ultrasonography

\section{METHODS}

This was a retrospective study performed at KK Women's and Children's Hospital, Singapore, the nation's largest tertiary-level public paediatric hospital. The hospital's electronic surgical records between 1 May 2008 and 30 June 2016 were searched for institutional codes of procedures describing open reduction of the hip. Paper surgical records from 1 July 2006 to 30 April 2008 were then searched for operative procedures involving at least an open reduction of the hip related to $\mathrm{DDH}$. All diagnoses of DDH was made by at least a senior specialist consultant with ample clinical experience.

Exclusion criteria were non-DDH hip dislocations, such as cerebral palsy, teratologic hip dislocation, fracture, tumour, septic arthritis, global developmental delay and slipped capital femoral epiphysis. Patients were grouped into those born at our institution (Group 1) and those born outside of our institution (Group 2).

Data collected included patient demographics, presenting complaint, birth location (within institution, national or international), comorbidities, risk factors of $\mathrm{DDH}$, whether there were any previous treatments and the presence of institutional newborn screening. Data was analysed qualitatively and quantitatively. Fisher's exact test was used to test for significance between the categorical samples, with the significance level set at $\mathrm{p}<0.05$. Ethical approval was obtained from the institutional review board before commencing the study.

\section{RESULTS}

Following exclusions, a total of 27 patients were included in the study (Fig. 2). Their mean age at presentation was $25 \pm 19$ months. All patients had unilateral DDH and for the majority ( $n=21,77.8 \%$ ), the site of involvement was on the left. Most of the patients $(n=22,81.5 \%)$ were female. $9(33.3 \%)$ patients had received prior treatment. The mean age at surgery was $40 \pm 31$ months. Open reduction of the hip was performed in all procedures. The most commonly performed procedure for open reduction was soft tissue release with acetabuloplasty $(\mathrm{n}=20,74.1 \%)$, followed by soft tissue release with proximal femur osteotomy and acetabuloplasty $(\mathrm{n}=5,18.5 \%$ ) and soft tissue release only $(n=2,7.4 \%)$. The most common presenting complaints for which patients were referred to our institution were limping $(\mathrm{n}=10,37.0 \%)$ and a recent diagnosis of $\mathrm{DDH}(\mathrm{n}=10$, $37.0 \%)$, followed by limb length discrepancy ( $n=5,18.5 \%$ ), delayed walking $(n=1,3.7 \%)$ and hip pain $(n=1,3.7 \%)$.

Group 1 had five patients and Group 2 had 22 patients (Table I). Of the 22 patients in Group 2, 11 patients were born outside the country of study. Both groups had similar age at 


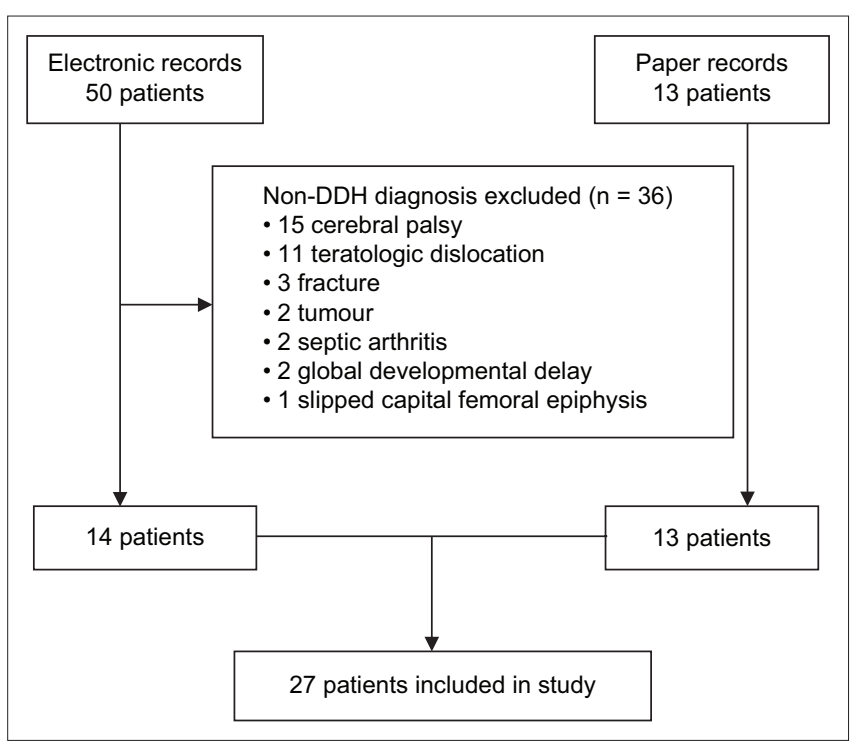

Fig. 2 Flowchart shows patient selection for the present study DDH: developmental dysplasia of the hip

presentation, gender distribution, site of involvement, number of risk factors and prior treatment. Both also had a large proportion of late presenters (Group 1: $n=4,80.0 \%$; Group 2: $n=21$, $95.5 \% ; p=0.34)$, defined as those presenting after three months of age. Patients in Group 1 had a significantly higher rate of documented newborn clinical screening (Group 1: $n=4,80.0 \%$ vs. Group 2: $n=1,4.5 \% ; p<0.01$ ).

In Group 1, one patient was screened to have DDH at birth and was commenced on the Pavlik harness. After the treatment failed, the patient underwent adductor release and closed reduction at three months of age, which also failed and required open reduction. A second patient was screened to be normal at birth and later presented at age 16 months with a limp. A third patient was screened to be normal at birth, but no proper documentation could be found. This patient later presented at age 48 months with limb length discrepancy. The last two patients had required immediate stay in the neonatal intensive care unit after birth due to respiratory distress and sepsis. It was therefore not possible to perform an immediate newborn examination for these two patients. They were only examined at the fifth and 31 st days of life, respectively, during which both patients were found to be normal by junior physicians.

\section{DISCUSSION}

This study showed that the main reason for performing open reductions for $\mathrm{DDH}$ is late presentation. This is consistent with Sanghrajka et al's study, ${ }^{(8)}$ in which the authors found that open reductions of the hip were performed mainly for late presentation rather than failure of early non-operative treatment. Limping and limb length discrepancy were the most common presenting complaints, which was similar to our study.

We postulate that one of the reasons for late presentation in $\mathrm{DDH}$ cases is the absence of institutionalised newborn clinical screening programmes. As shown in this study, only one out of 22 patients born outside of our institution was screened as a newborn. The prevalence of late diagnosis can
Table I. Patient characteristics according to place of birth.

\begin{tabular}{|llll|}
\hline \multirow{2}{*}{ Characteristic } & \multicolumn{2}{c}{ No. (\%) } & p-value \\
\cline { 2 - 4 } & $\begin{array}{l}\text { Group 1 } \\
\text { (n= 5) }\end{array}$ & $\begin{array}{l}\text { Group 2 } \\
\text { (n= 22) }\end{array}$ \\
\hline Age at presentation* (mth) & $16(0-48)$ & $18(1-80)$ & 0.68 \\
\hline Late presentation & $4(80.0)$ & $21(95.5)$ & 0.34 \\
\hline Screened at birth & $4(80.0)$ & $1(4.5)$ & $<0.01$ \\
\hline Female gender & $3(60.0)$ & $19(86.4)$ & 0.22 \\
\hline Age at surgery* $(m+h)$ & $24(19-52)$ & $28(11-159)$ & 0.85 \\
\hline Left hip & $5(100.0)$ & $16(72.7)$ & 0.56 \\
\hline Previous treatment & $1(20.0)$ & $8(36.4)$ & 0.64 \\
\hline$\geq 1$ risk factor & $4(80.0)$ & $20(90.9)$ & 0.47 \\
\hline
\end{tabular}

*Data presented as median (range). Group 1: born at institution; Group 2: born outside of institution

be as low as $2.4 \%$ in the presence of a screening programme, based on clinical examination alone. ${ }^{(7)}$ Therefore, we advocate institutionalised newborn clinical screening programmes at all maternity centres.

Even so, the success of such screening programmes would depend almost entirely on their quality. For instance, after newborn screening was first introduced in the United Kingdom, $70 \%$ of DDH patients were still diagnosed late (defined as after age three months). ${ }^{(9)}$ Similarly, $70 \%$ of DDH patients were diagnosed only after the age of three months in Northern Ireland. ${ }^{(10)}$ The initial newborn examination is thus paramount to preventing the late diagnosis of $\mathrm{DDH}$. It is known that the examiner's experience in clinical examination is important for picking up early signs of $\mathrm{DDH} .{ }^{(11)}$ This was evident in our study, where there were four patients from our institution with a missed diagnosis, all of whom were not examined by a senior specialist physician during the newborn clinical screening. We recommend that all newborn clinical screenings be re-examined by a physician of at least a senior specialist level if the initial examination had been performed by a junior.

There were a few limitations to our study. Firstly, it was retrospective in nature. Secondly, the 'developmental' nature of $\mathrm{DDH}$ means that some newborns were inevitably correctly screened to be normal but developed DDH later in life. ${ }^{(12)} \mathrm{A}$ safety net should be in place to detect these patients (e.g. active review by a general practitioner or health visitor at specified intervals after birth, such as the system in place in Northern Ireland). ${ }^{(10)}$ Thirdly, this was a single-institution study, so the true rate of open reduction for late DDH diagnosis in Singapore is not known. However, our findings may still be representative of the local patient population, as our institution is the largest of only two public paediatric hospitals in the country; in addition, it has been suggested elsewhere that the rate of late DDH diagnosis is similar between private and public sector hospitals. ${ }^{(13)}$

In conclusion, the lack of institutionalised newborn clinical screening appears to be the root cause of late presentation of DDH leading to open surgery. We recommend that all maternity units put in place an institutionalised newborn clinical screening programme for $\mathrm{DDH}$. The examining physician should be experienced and of at least senior specialist level. 


\section{REFERENCES}

1. Lisle R, Boekelaar M, Stannage K, Whitewood C. Delayed diagnosis of developmental dislocation of the hip: the Western Australian experience. ANZJ Surg 2012; 82:612-5

2. Ortolani M. Un segno poco noto e sua importanza per la diagnosis precoce $\mathrm{di}$ prelussazione conenita dell'anca. Pediatric 1937; 45:129-36.

3. Barlow TG. Early diagnosis and treatment of congenital dislocation of the hip. Proc R Soc Med 1963; 56:804-6.

4. Lotito FM, Rabbaglietti G, Notarantonio M. The ultrasonographic image of the infant hip affected by developmental dysplasia with a positive Ortolani's sign. Pediatr Radiol 2002; 32:418-22.

5. Witt C. Detecting developmental dysplasia of the hip. Adv Neonatal Care 2003; 3:65-75.

6. Tomlinson J, O'Dowd D, Fernandes JA. Managing developmental dysplasia of the hip. Indian J Pediatr 2016; 83:1275-9.

7. Chan A, Cundy PJ, Foster BK, Keane RJ, Byron-Scott R. Late diagnosis of congenital dislocation of the hip and presence of a screening programme: South Australian population-based study. Lancet 1999; 354:1514-7.
8. Sanghrajka AP, Murnaghan CF, Shekkeris A, Eastwood DM. Open reduction for developmental dysplasia of the hip: failures of screening or failures of treatment? Ann R Coll Surg Engl 2013; 95:113-7.

9. Godward S, Dezateux C. Surgery for congenital dislocation of the hip in the UK as a measure of outcome of screening. MRC Working Party on Congenital Dislocation of the Hip. Medical Research Council. Lancet 1998; 351:1149-52.

10. Donnelly KJ, Chan KW, Cosgrove AP. Delayed diagnosis of developmental dysplasia of the hip in Northern Ireland: can we do better? Bone Joint J 2015; 97-B:1572-6.

11. Finne PH, Dalen I, Ikonomou N, Ulimoen G, Hansen TW. Diagnosis of congenital hip dysplasia in the newborn. Acta Orthop 2008; 79:313-20.

12. Kotlarsky P, Haber R, Bialik V, Eidelman M. Developmental dysplasia of the hip: what has changed in the last 20 years? World J Orthop 2015; 6:886-901.

13. Morin C, Wicart P; French Society of Pediatric Orthopaedics. Congenital dislocation of the hip, with late diagnosis after 1 year of age: update and management. Orthop Traumatol Surg Res 2012; 98(6 Suppl):S154-8. 